These results are a preliminary account of comprehensive investigations at present being undertaken with respect to type of wave, range of materials, magnetic field-strength and frequency of the pulse. Velocity measurements are also being correlated with the observations on attenuation.

Thanks are due to Sir George Thomson for providing facilities for this work in the Physics Depart. ment of the Imperial College.

Physics Department,

$$
\text { J. DE KLERK }
$$

Imperial College of Science and Technology, London, S.W.7. July 3.

${ }^{1} J$. App. Phys., 21, 10, 1087 (1950).

\section{A New Surface Viscometer}

OF the methods which have been used to measure the viscosity of surface films on a liquid substrate only one, the canal or surface-slit method, is amenable to rigorous mathematical treatment. Harkins and Kirkwood $^{1}$ derived an equation which could be applied to this method provided that certain modifications could be made to the apparatus as used by $\mathrm{Joly}^{2}$. To measure the viscosity of dilute soluble films (to which the surface-slit method cannot be applied), a new method has been developed which is capable of rigorous interpretation.

The substrate (water or solution) is made to flow at a constant volume rate, $V$, through a horizontal channel of rectangular cross-section which it fills completely. The velocity, $v_{m}$, of an element of the surface along the centre of the channel may be measured by observing the passage of a floating particle of graphite between marked positions.

So long as there is no surface-pressure gradient, the flow velocity, $v$, satisfies, besides the NavierStokes equation, the boundary condition in the surface $(z=0)$ :

$$
\mu \partial^{2} v / \partial x^{2}+\eta \partial v / \partial z=0
$$

where the distance, $x$, is measured across the channel and $z$ vertically downward. For a channel of width $a$ and depth $b$ and a very small hydrostatic pressure gradient, $A$, in the direction of flow we find :

$v_{m}=\frac{4 A a^{3}}{\pi^{3}} \Sigma^{\prime} \sin \frac{n \pi}{2} \frac{\tanh (n \pi b / 2 a)}{n^{3} a \eta \operatorname{coth}(n \pi b / a)+n^{4} \pi \mu}$

and

$$
V=\frac{A b a^{3}}{12 \eta}=\frac{8 A a^{4}}{\eta \pi^{5}} \Sigma^{\prime} \frac{a \eta+2 n \pi \mu \tanh (n \pi b / 2 a)}{n^{5} a \eta+n^{6} \pi \mu \operatorname{coth}(n \pi b / 2 a)},
$$

where the sum is to be taken over all odd values of $n$. The ratio $v_{m} / V$ is independent of $A$, and for $b \geqslant 3 a$ simplifies to:

$$
\frac{v_{m}}{V}=\frac{48 \Sigma^{\prime}(-1)^{(n-1) / 2} \frac{1}{n^{3}+n^{4} \pi \mu / a \eta}}{\pi^{3} a b-\frac{96 a^{2}}{\pi^{2}} \Sigma^{\prime} \frac{1}{n^{5}} \frac{a \eta+2 n \pi \mu}{a \eta+n \pi \mu}}
$$

The only unknown in this equation is $\mu$; for given values of $a, b$ and $\eta$, it is a simple matter to plot the ratio $v_{m} / V$ against $\mu$. The measurement of $v_{m}$, to give less than 5 per cent error, must be confined to particles floating along the central 20 per cent of the channel surface.

To illustrate the sensitivity of the method, for $a=0.153 \mathrm{~cm} ., b=0.60 \mathrm{~cm}$., and $\eta=0.01$ poise, the following table shows values of $v_{m} / V$ corresponding to a range of values of $\mu$ :

$\begin{array}{lccccccc}\mu \times 10^{4} \text { (surface poise) } & 0 & 0 \cdot 5 & 1 & 2 & 5 & 10 & 20 \\ v_{m} / V & 17 \cdot 8 & 16 \cdot 3 & 15 \cdot 1 & 13 \cdot 1 & 9 \cdot 3 & 6 \cdot 3 & 3 \cdot 8\end{array}$

An experimental difficulty occurs in measuring the surface viscosity of some surface-active solutions. A negative surface-pressure gradient is set up along the channel, tending to retard the surface flow. If a barrier is placed across the surface of the channel before commencing the bulk flow, the surface pressures at the ends of the channel may be kept the same. When the flow has reached a steady state, the barrier is lifted and measurements of $i_{m}$ are made after measured intervals. The value of $v_{m}$ for zero surfacepressure gradient is then obtained by extrapolation. The form of the $v_{m}$ versus time curve, and the diffi. culty of obtaining measurements at short intervals after lifting the barrier, sometimes makes this method impracticable and recourse is being made to a second method. After a period, $v_{m}$ approaches a constant value, apparently indicating that a steady state with a constant surface-pressure gradient has been set up. By making further assumptions it is possible to make allowance for this back pressure (a measurable quantity) and to calculate the surface viscosity from the steady-state flow.

It must be assumed, with Harkins and Kirkwood ${ }^{1}$, that the surface film is incompressible, that surface viscosity is independent of surface pressure and that there is no slip between surface film and substrate. Equations for $v_{m}$ and $V$, obtained in the same way as the Harkins and Kirkwood equation in terms of a surface-pressure drop $B$, may be superposed linearly on the equations (1) and (2) to give equations for the flow when both hydrostatic and surface-pressure gradients are operative. The hydrostatic pressure gradient, $A$, is again eliminated between these equations to obtain an equation of the form :

$$
V K_{1}+B K_{2}-v_{m} K_{3}=0,
$$

where $K_{1}, K_{2}$ and $K_{3}$ are analytical functions of $\mu, \eta, a$ and $b$. By plotting $v_{m} / V$ against $\mu$, a family of curves is obtained for various values of $B / V$.

Where the surface film is moderately close packed, for example, in condensed insoluble films and in concentrated soluble films, a back pressure develops so rapidly that extrapolation to zero back pressure is impossible and $v_{m}$ becomes so small that accurate determination of its steady value becomes impracticable. This constitutes a major limitation on our method of measuring surface viscosity. For dilute gaseous films, soluble or insoluble, the method appears most promising.

A more complete description of the experimental methods and the mathematical treatment will be published later.

Division of Industrial Chemistry,

Commonwealth Scientific and

Industrial Research Organization, Melbourne.

W. E. Ewers

Division of Electrotechnology,

Commonwealth Scientific and

Industrial Research Organization, Sydney.

June 26.

${ }^{1}$ Harkins, W. D., and Kirkwood, J. G., J. Chem. Phys., 6, 53 (1938). 2 Joly, M., J. Phys. et Rad., 8, 471 (1937). 